\title{
Setup for Triggering Force Testing of Touch Probes for CNC Machine Tools and CMMs
}

\author{
A. Wozniak, M. Byszewski, M. Jankowski \\ Institute of Metrology and Biomedical Engineering, Faculty of Mechatronics, Warsaw University of Technology, Sw. A. \\ Boboli 8 Street, 02-525 Warsaw, Poland, wozniaka@mchtr.pw.edu.pl, mbyszewski@gmail.com, \\ M.Jankowski@mchtr.pw.edu.pl
}

\begin{abstract}
Touch-trigger probes are commonly used both in coordinate measuring machines (CMM) and in computer numerical control (CNC) machine tools. In both cases accuracy of measurement of the overall system and probing unit are closely interrelated. Key parameters of the probes are repeatability and pre-travel variation dependent on adjustable stylus force. To enable testing of the triggering force of the probes, the new setup was developed. The principle of the method and set-up is presented and its validity is experimentally confirmed.
\end{abstract}

Keywords: Triggering force, touch-trigger probe, coordinate measuring machines, on-machine measurement

\section{INTRODUCTION}

$\boldsymbol{F}^{\mathrm{O}}$ OR A LONG TIME, the contact probes have been in common use in coordinate measuring machines (CMM) for localizing the points on the surface of the measured object in the machine's measuring space. In recent years, the possibility of an on-machine measurement with a touchtrigger probe of an object directly on a $\mathrm{CNC}$ machine tool became widespread. This eliminates the necessity to transport an object to a CMM and of a repeated positioning of the object on the CNC machine tool in order to correct the detected defects. Inspection probes are mounted in the working center spindle or in the CNC lathe's revolver head and they are used to set the object cutting and its initial dimension control. In the case of inspection probes, mount on the tool's spindle wireless transfer is commonly used: optical in the infrared band or radio transfer.

\section{TRIGGERING FORCE OF TOUCH-TRIGGER PROBES}

In the case of CMMs as well as of CNC machine tools, the key parameters are the performance parameters, such as repeatability and pre-travel variation. That is why methods of determining those parameters were developed and tests of performance of various probes were conducted. Most of this research is on the subject of CMM probes [1]-[6], but lately articles on probes used in CNC machine tools appeared [7][11]. In order to compensate probe systematic errors, theoretical models describing their operation and errors were developed [12]-[18]. As for now, all the models developed apply only to probes used in CMMs. Those existing probe working models take under account the value of force operating on the tip of the stylus necessary to trigger the probe, depending on its operation direction. This way, elastic deflection of the stylus in the moment of triggering of the probe, depending on the operation direction, is determined. The variation of those deflections is the main source of probe errors. Determining the value of the triggering force in the function of the measurement direction by the user is, unfortunately, complicated, and until now no such simple procedure exists. The little news on this topic is not entirely satisfactory. In the work of Aston et al. [14] experimental methods of testing the triggering force of a touch-trigger probe TP2 in the XY plane for three lengths of styli were described. They were built with a gram gauge produced by Renishaw. The triggering of the probe was forced by the machine movement in the $\mathrm{X}$ axis, and different directions of probe work were obtained by using an articulating head PH9. The Renishaw gram gauge was also used by Cauchick-Miguel et al. to acquire the planar triggering force characteristic [3]. Woody and Smith described a new type of probe they constructed in their paper [19]. They also proposed a setup for testing the triggering force, which enabled investigation of the response of a new probe to a given force acting in a given direction. They describe a setup where the probe is mounting mounted to an arrangement of rotating stages, which allows its angular positioning in regard to a piezoelectric actuator equipped with a force transducer. The displacement of the actuator is measured with a capacitive sensor. The force transducer is built of two elastically bound beams. Their reciprocal displacement is measured with a capacitive sensor. Such a setup enables testing of very low forces (up to $0.03 \mathrm{~N}$ ) and cannot be employed for testing probes used in machine tools, where the triggering force may be over $10 \mathrm{~N}$.

Likewise, a device was described in the work of Park et al. [20], which enabled testing of a reaction of another developed touch-trigger probe under a given force, or of a given displacement of a stylus. For this device, a known mass up to $1 \mathrm{~N}$ is used to give the force. The direction of the force or of the displacement is determined according to the rotation of the probe in two axes.

Unfortunately, the described methods of investigating the triggering force are insufficient, especially in the case of the probes used in CNC machine tools. That is why further work on developing such stands is necessary.

Apart from probing errors of touch-trigger probes, there are many other sources of the coordinate measurement uncertainty: geometric errors of the CMM or the CNC machine, influence of the temperature, inaccuracy of the used software and so on. Also touch-trigger probes are not the only type of probes that can be used. In CMMs there are 
widely used scanning probes. These probes not only detect the contact of the stylus tip with the measured surface, but also continuously measure the displacement of the stylus tip, so the scanning (measurement without breaking contact between the stylus tip and the measured surface) is possible. This type of measurement is faster than measurement with touch-trigger probes, but the results are affected by dynamic errors of the machine. The above mentioned issues were described in [21-24].

The use of scanning probes is not popular in $\mathrm{CNC}$ machines, but it may change in the future.

The developed setup enables testing of the triggering force in most types of touch-trigger probes used in CNC machine tools as well as in CMMs. The constructions, as well as examples of results obtained with this setup are presented in the article.

\section{NEW SETUP FOR TESTING THE TRIGGERING FORCE}

A diagram of the setup is presented in Fig.1, and its mechanical portion is presented in Fig.2.

A (1) FSG series Honeywell force sensor of a range between 0 to $14.7 \mathrm{~N}$ was used for the setup construction. The sensor is moved by a piezoelectric actuator (3) of a 90 $\mu \mathrm{m}$ stroke movement range. The other units are: force measurement control unit, displacement measurement control unit, probe and actuator positioning units and the probe interface unit. The force measurement sensor does not touch the tip of the probe (2) before the measurement. The measurement starts by resetting the force sensor's indication in the given position. Next, the piezoelectric actuator (3) is moved towards the probe's stylus tip, which results in the touching of the force sensor with the stylus tip, and, afterwards, the styli's tilt and the triggering of the probe. The value of the force operating on the sensor while it triggers the probe is recorded and transferred to the PC. The PC is used to control the measurement and to acquire data. To minimize the effect of environmental disturbances on the measurements result, the measurement signal emitted by the force sensor is amplified by the unit (4), situated near the sensor. The sensor's working direction can be set by rotating the probe around its axis, and by rotating the piezoelectric actuator around the axis perpendicular to the probe's axis. The probe fixing and positioning unit as well as the piezoelectric actuator fixing and positioning unit also enable moving those elements along their axes. This enables placing the stylus tip in the piezoelectric actuator's axis and ensures the distance between the force sensor in its initial position and the stylus tip is correct.

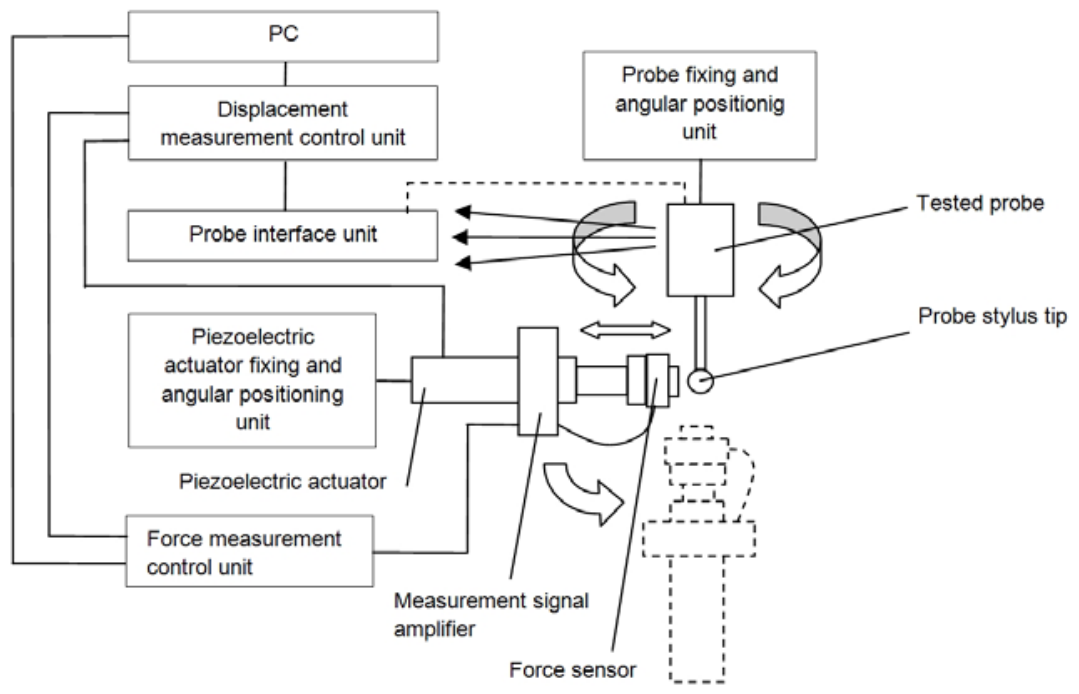

Fig.1. A scheme of the measurement setup

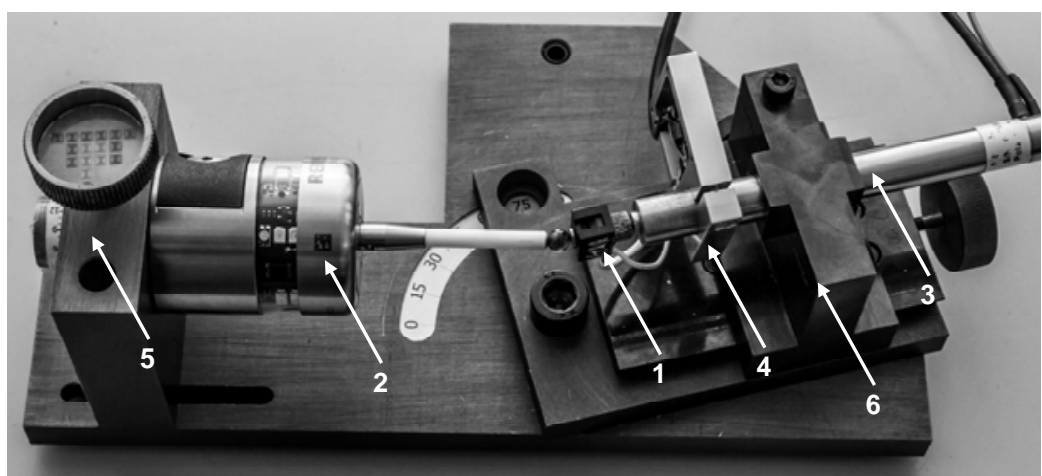

Fig.2. Mechanical portion for testing the probe triggering force: 1 - force sensor, 2 - the probe, 3 - piezoelectric actuator, 4 - measurement signal amplifying unit, 5 - probe fixing and angular positioning unit, 6 - piezoelectric actuator fixing and angular positioning unit 


\section{CALIBRATION OF THE SETUP AND DETERMINATION OF THE MEASUREMENT UNCERTAINTY}

The setup was calibrated with M1 test weights, mass ranging from $5 \mathrm{~g}$ to $500 \mathrm{~g}$. The maximum permissible mass error for this class of weights ranging to $500 \mathrm{~g}$ is $\pm 0.025 \mathrm{~g}$, and therefore much inferior to the setup's resolution which is $1 \mathrm{~g} .50$ measurements were run for each weight. Afterwards, a linear regression equation was determined. This equation describes the dependence between the weight acting on the sensor and the sensor's indication. The obtained parameter $\mathrm{R}^{2}$, which describes the level of conformity with the linear model, is 0.998 .

The results obtained during calibration are shown in Fig.3.

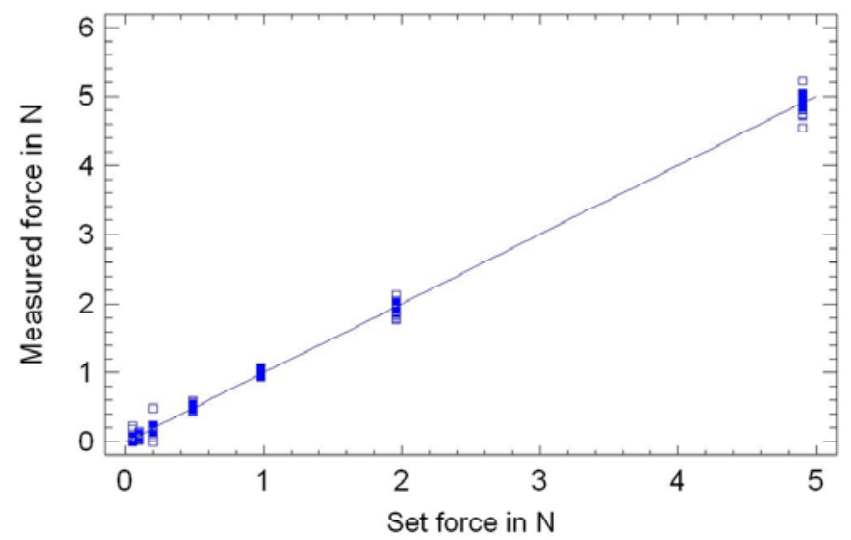

Fig.3. Results obtained during the force sensor calibration.

The results confirm the linear characteristic of the post's indications. No systematic errors occurred. That is why the estimate of the force measurement uncertainty was based on the indication spread.

To obtain it, the distribution of the results obtained during calibration for each load level was tested. Statistical tests show that on the confidence level of $95 \%$ we can reject the hypothesis that it is the result of normal distribution. That is why the value of range $R$ was used as the value of the result dispersion. The maximum value of the range $R$ shown by the post during calibration is $0.68 \mathrm{~N}$.

Therefore, the standard force measurement uncertainty $u(F)$ is:

$$
\mathrm{u}(\mathrm{F})=\frac{\mathrm{R}}{2 \sqrt{3}}=0.2 \mathrm{~N}
$$

Assuming a coverage factor of $\mathrm{k}=2$ we obtain the expanded uncertainty of the force measurement $U(F)=0.4$ $\mathrm{N}$.

The influence of random errors can be reduced by performing a series of $\mathrm{n}$ measurements and calculating an average of obtained results. Therefore:

$$
U(\bar{F})=\frac{U(F)}{\sqrt{n}}
$$

Hence, for the series of 50 measurements the measurement uncertainty is $0.06 \mathrm{~N}$. During the calibrating, the largest deviation of the average force value from the given force value was not higher than $0.1 \mathrm{~N}$.

\section{RESULTS OF TESTING THE PROBE TRIGGERING FORCE}

To demonstrate the measurement possibilities of the proposed setup, tests of spatial characteristics of force were run on two types of probes: the TP6 and the OMP40-2 probe. The TP6 probe is a kinematic probe used in CMMs The OMP40-2 with an OMI interface is a probe designated for $\mathrm{CNC}$ machine tools. A test of the triggering force for the azimuth angles $\alpha$ ranging from $0^{\circ}$ to $350^{\circ}$ with a step of $10^{\circ}$, and for the latitude angles $\beta 0^{\circ}$ to $90^{\circ}$ with a step of $15^{\circ}$ was performed. For each measuring direction a series of 50 measurements was held. In Figs. 4 and 5 spatial diagrams of an average triggering force for each direction, respectively for the TP6 and OMP40-2 probes are shown. In order to compare the results, the diagrams were presented in the same scale. As expected, the triggering force values for the OMP40-2 probe are superior to the respective values for the TP6 probe. For both examined probes, the triggering force is the greatest in axial direction ( 2.48 for the TP6 probe and $5.89 \mathrm{~N}$ for the OMP-40-2 probe, respectively). That is because this and similar directions of the triggering of the probe requires translation, instead of tilting, of a movable element of the transducer. For all the other tested work directions and for both tested probes the radial element of the triggering force is only slightly dependent of the latitude angle $\beta$, but as this angle increases, the axial element of the triggering force increases too. This is because the radial element of the force acting on the stylus tip has larger impact on the torque causing the tilt of the movable element of the kinematic transducer, as the length of its lever arm is superior to the length of the lever arm of the axial component of the force.

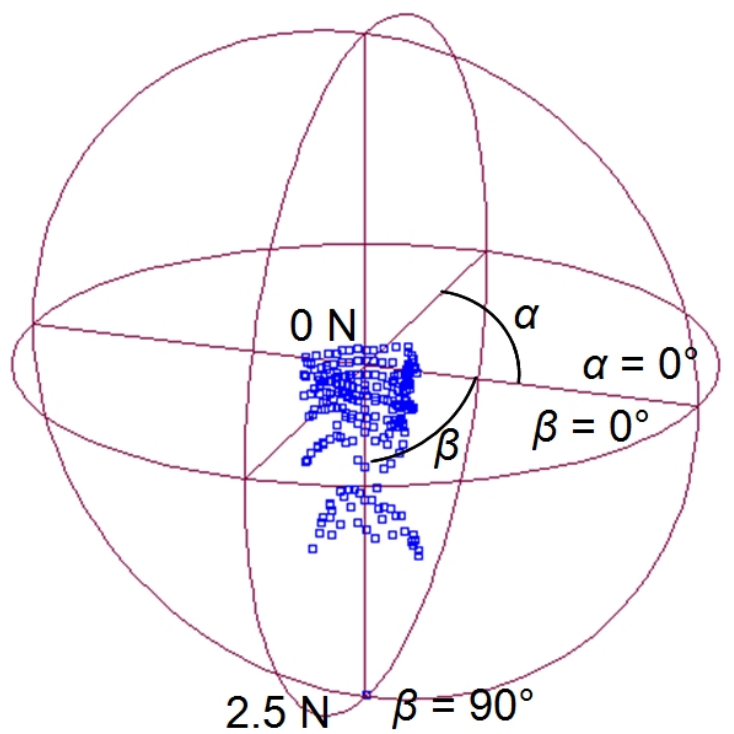

Fig.4. Spatial diagram of the triggering force for the TP6 probe 


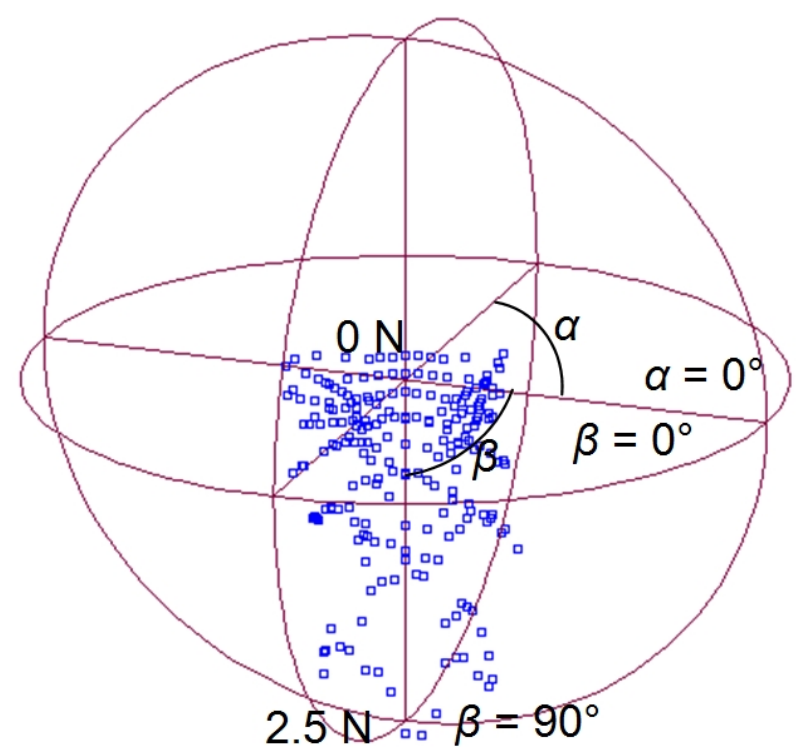

Fig.5. Spatial diagram of the triggering force for the OMP40-2 probe. The value of the triggering probe for the axial direction, which is $5.89 \mathrm{~N}$, is not shown on the diagram

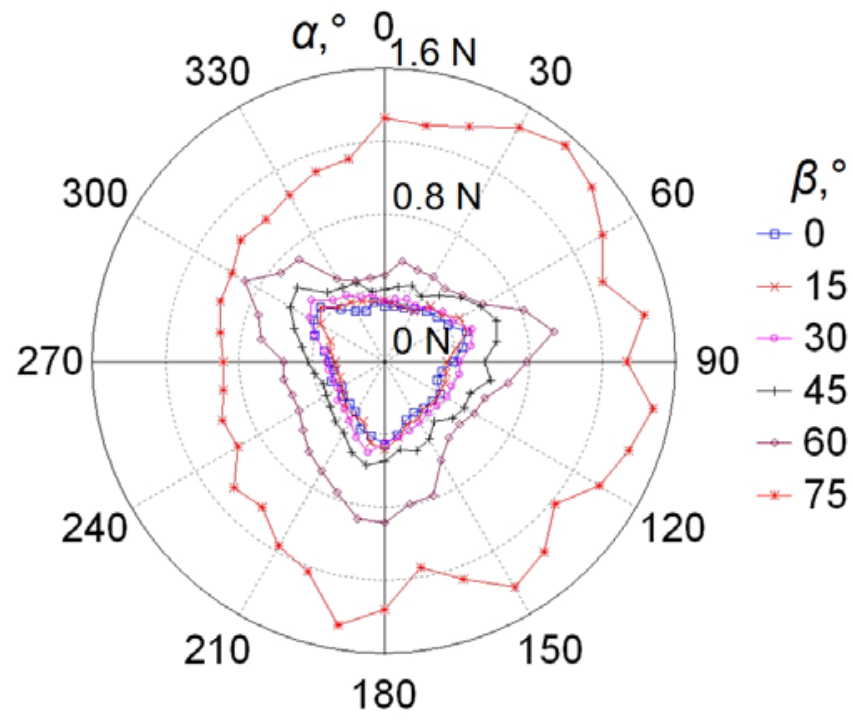

Fig.6. The diagram of the average triggering force for the TP6 probe

The two-dimensional diagrams of the average triggering force for each $\beta$ angle, for TP6 and OMP40-2 probes, respectively, are shown in Fig.6 and Fig.7.The force value of triggering for the axial direction is not shown in those diagrams. Because of the differences in the triggering force values for the tested probes, the diagrams are shown in different scales.

The obtained results show that for small latitude angles the characteristics of the triggering force have a triangular shape. This remains in accordance with theoretical models, according to which triggering of the probe is in most cases caused by the rotation of the movable element of the kinematic transducer around the axis containing two fulcrums. In such a case, the only important component of the force is the component situated in the plane perpendicular to the abovementioned axis. The lowest average triggering force value determined for the TP6 probe is $0.26 \mathrm{~N}$. Hence, the measurement uncertainty of the force for all the directions of the probe work is not at least five times inferior to the investigated value.

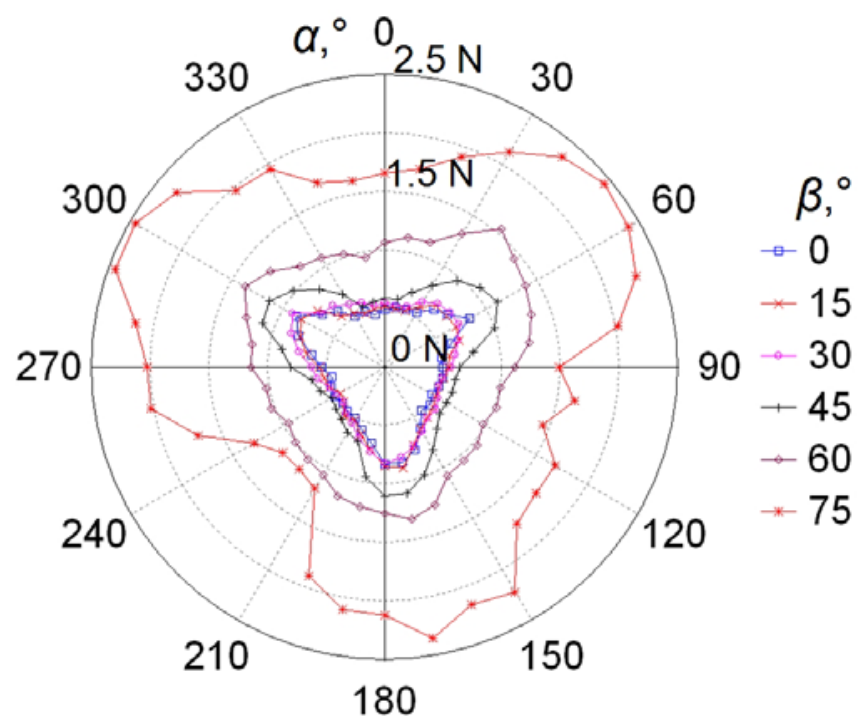

Fig.7. The diagram of the average triggering force for the OMP40-2 probe

In case of the OMP40-2 probe, the minimal determined average triggering force value is $0.44 \mathrm{~N}$, and therefore the measurement uncertainty is 5 times inferior to the lowest investigated force value. The triggering force value increases with the latitude angle value.

The larger is the latitude angle, the larger is the triggering force. This is because, as it was written above, the length of the lever arm of the radial component of the force component is superior to the length of the lever arm of the axial component of the force.

\section{CONCLUSIONS}

The article presents the idea and the principle of operation of a new setup for testing the spatial characteristics of the triggering force of the touch-trigger probes used in CMMs and CNC machine tools. The setup was calibrated. The expanded measurement uncertainty for a single measurement of the triggering force is $0.4 \mathrm{~N}$. A measurement uncertainty of an average triggering force for a series of 50 measurements is $0.06 \mathrm{~N}$. The validity of the setup was tested both for a TP6 probe used in CMMs, and for an OMP40-2 probe used in CNC machine tools. Spatial characteristics were obtained. A quality evaluation shows that their shape is in accordance with the expectations derived from the existing theoretical models. This confirms the setup's utility for users as well as for producers of probes used in CMMs and CNC machine tools.

We suspect that improving the setup electronics, for example ensuring more stable power voltage, would improve the setup accuracy. Also, automation of the probe and piezotranslator rotations would allow executing measurements much quicker. Thus, future works are necessary. 


\section{ACKNOWLEDGMENT}

Research co-funded from the research budget for 20102013 as a research project of The National Centre for Research and Development.

\section{REFERENCES}

[1] Cauchick-Miguel, P., King, T., Abackerli, A. (1998). A review on methods for probe performance verification. Measurement, 23 (1), 15-33.

[2] Chan, F.M.M., Davis, E.J., King, T.G., Stout, K.J. (1997). Some performance characteristics of a multiaxis touch trigger probe. Measurement Science and Technology, 8 (8), 837-848.

[3] Cauchick-Miguel, P.A., King, T., Abackerli, A.J. (2003). CMM touch trigger performance verification using a probe test apparatus. Journal of the Brazilian Society of Mechanical Sciences and Engineering, 25 (2).

[4] Cauchick-Miguel, P.A., King, T.G. (1998). Factors which influence $\mathrm{CMM}$ touch trigger probe performance. International Journal of Machine Tools \& Manufacture, 38 (4), 363-374.

[5] Dobosz, M., Woźniak, A. (2005). CMM touch trigger probes testing using a reference axis. Precision Engineering, 29 (3), 281-289.

[6] Salah, H.R.A. (2010). Probing system characteristics in coordinate metrology. Measurement Science Review, 10 (4), 120-129.

[7] Cho, M.W., Seo, T.I. (2002). Machining error compensation using radial basis function network based on $\mathrm{CAD} / \mathrm{CAM} / \mathrm{CAI}$ integration concept. International Journal of Production Research, 40 (9), 2159-2174.

[8] Cho, M.W., Seo, T.I. (2002). Inspection planning strategy for the on-machine measurement process based on $\mathrm{CAD} / \mathrm{CAM} / \mathrm{CAI}$ integration. International Journal of Advanced Manufacturing Technology, 19 (8), 607-617.

[9] Cho, M.W., Seo, T.I., Kwon, H.D. (2003). Integrated error compensation method using OMM system for profile milling operation. Journal of Materials Processing Technology, 136 (1-3), 88-99.

[10] Choi, J.P., Min, B.K., Lee, S.J. (2004). Reduction of machining errors of a three-axis machine tool by onmachine measurement and error compensation system. Journal of Materials Processing Technology, 155-156 (1-3), 2056-2064.

[11] Zeleny, J., Janda, M. (2009). Automatic on-machine measurement of complex parts. Modern Machinery Science Journal, 2 (1), 92-95.

[12] Estler, W.T., Phillips, S.D., Borchardt, B., Hopp, T., Witzgall, C., Levenson, M., Eberhardt, K., McClain, M., Shen, Y., Zhang, X. (1996). Error compensation for CMM touch trigger probes. Precision Engineering, 19 (2-3), 84-96.

[13] Mayer, J.R.R., Ghazzar, A., Rossy, O. (1996). 3D characterisation, modelling and compensation of the pre-travel of a kinematic touch trigger probe. Measurement, 19 (2), 83-94.

[14] Aston, R.A.E., Davis, J., Stout, K.J. (1997). Forced touch-down: A customer's further examination of a touch trigger probe. International Journal of Machine Tools \& Manufacture, 37 (10), 1383-1392.

[15] Estler, W.T., Phillips, S.D., Borchardt, B., Hopp, T., Levenson, M., Eberhardt, K., McClain, M., Shen, Y., Zhang, X. (1997). Practical aspects of touch-trigger probe error compensation. Precision Engineering, 21 (1), 1-17.

[16] Shen, Y.-L., Zhang, X. (1997). Modelling of pretravel for touch trigger probes on indexable probe heads on coordinate measuring machines. International Journal of Advanced Manufacturing Technology, 13 (3), 206213.

[17] Woźniak, A., Dobosz, M. (2003). Metrological feasibilities of CMM touch trigger probes. Part I: 3D theoretical model of probe pretravel. Measurement, 34 (4), 273-286.

[18] Woźniak, A. (2006). Simple method of 3D error compensation of triggering probes on coordinate measuring machine. Metrology and Measurement Systems, 13 (3), 289-302.

[19] Woody, S.C., Smith, S.T. (2003). Resonance-based vector touch sensors. Precision Engineering, 27 (3), 221-233.

[20] Park, J.J., Kwon, K., Cho, N. (2006). Development of a coordinate measuring machine (CMM) touch probe using a multi-axis force sensor. Measurement Science and Technology, 17 (9), 2380-2386.

[21] Schwenke, H., Knapp, W., Haitjema, H., Weckenmann, A., Schmitt, R., Delbressine, F. (2008). Geometric error measurement and compensation of machines-An update. CIRP Annals - Manufacturing Technology, 57 (2), 660-675.

[22] Farooqui, S.A., Morse, E.P. (2007). Methods and artifacts for comparison of scanning CMM performance. Journal of Computing and Information Science in Engineering, 7 (1), 72-80.

[23] Kosarevsky, S., Latypov V. (2010). Inertia compensation while scanning screw threads on coordinate measuring machines. Measurement Science Review, 10 (2), 68-71.

[24] Hessling, J.P. (2008). Dynamic metrology-an approach to dynamic evaluation of linear timeinvariant measurement systems. Measurement Science and Technology, 19 (8), 084008.

Received July 2, 2012. Accepted January 28, 2013. 